\title{
HLA Haplotypes in Dizygotic Twin Pairs: Are Dizygotic Twins More Similar Than Sibs?
}

\author{
Ingrid Louise Titlestad', Kirsten Ohm Kyvik², Tom Kristensen', Søren Lillevang' \\ 'Department of Clinical Immunology, Odense University Hospital, Denmark; \\ ${ }^{2}$ Department of Genetic Epidemology, Institute of Community Health, University of Southern Denmark, Denmark.
}

\begin{abstract}
t has been suggested that dizygotic twin pairs share two HLA haplotypes more often than ordinary siblings and thus might be genetically more alike. We tested this hypothesis in dizygotic twin pairs from the Danish Twin Registry. A total of 114 (60 female and 54 male) same-sexed healthy twin pairs aged $18-45$ years participated. Dizygosity was established by means of DNA sequencing of nine polymorphic markers. HLA-A, B and $\mathrm{CW}$ specificities were typed with serology, and if data were inconclusive, with DNA typing. If twin partners had the same HLA-types, they were assumed to share two haplotypes. If they had 1 HLA A, B and $C$ antigen group in common they were assumed to share one haplotype and if they had no HLA types in common they were assumed to share zero haplotypes. Since HLA-types from parents were unavailable we could not test for identity-by-descent and thus had a risk of overestimating the number of twins sharing two haplotypes. A Chi-square test was used to compare observed numbers in each haplotype sharing group with the expected numbers. Twenty-nine (expected 28.5) twin pairs had two HLA-types in common, 52 (expected 57) had one HLA-type in common and 33 (expected 28.5) had zero HLA-types in common, $p=0.56$. Our data show that DZ twins are not more similar than sibs from different pregnancies in general.
\end{abstract}

Twin studies have been commonly used as a research tool in genetic epidemiology and medical genetics to establish whether phenotypes have a genetic component to their ethiology (e.g., insulin dependent diabetes [IDDM], rheumatoid arthritis [RA], systemic lupus erythematosus [SLE] and schizophrenia). These studies of categorical disease phenotypes for example involve comparison of concordance among monozygotic (MZ) and dizygotic (DZ) twins, where a higher level of concordance between MZ twins compared to DZ twins support a genetic contribution. This comparison can be made on the priori assumption that DZ twins are genetically no more similar than sibs, having a Mendelian distribution of haplotype sharing: (i.e., $2=25 \%, 1=50 \%$ and $0=25 \%$ ).

Some groups have reported a trend towards increased sharing of HLA-haplotypes, suggesting that DZ twins are in general more genetically similar at the HLA system than sibs born from separate pregnancies (Jawaheer et al., 1996; Staub Nielsen et al., 1990). Increased sharing of HLA-haplotypes in DZ twins could be due to either prezygotic, zygotic or postzygotic selection.

We have HLA-typed $114 \mathrm{DZ}$ twin pairs of identical sex (60 female and 54 male) enrolled through the Danish Twin Register (Kyvik et al., 1995), which is a population-based register. We have only been able to evaluate HLA-pheno- types, since data from the parents were not available, and thus genotypes and linkage phases could not be deduced.

\section{Method \\ Participants}

Twins in the Danish Twin Register were invited to participate in several parallel projects as a panel approved by the Danish Scientific-Ethical Committees (97/25 PMC). The criteria for invitation were age between 18 and 45 years and both twins should be healthy. Exclusion criteria were chronic diseases such as diabetes mellitus, rheumatoid arthritis, and chronic bowel infection These data were obtained from a questionnaire answered in 1994. After consent the twins donated blood samples on the same day.

\section{Zygosity}

Zygosity of twins was established independently by The Danish Twin Registry using standardized questionnaire and by Deoxyribonucleic acid- (DNA-) sequencing of nine polymorphic short tandem repeat systems with the PE Applied Biosystems AmpFISTR Profiler Plus Kit (D3S1358, HumVWA31/A(p9), FGA, D8S1179, D21S11, D18S51, D5S818, D13S317, D7S820) (Perkin-Elmer Corp. 1997 Users manual. Foster City: Perkin-Elmer Corp).

\section{HLA Typing}

All subjects were typed for HLA-A and -B (and -Cw) specificities by serology, using complement dependent cytotoxicity microassay (Lymphotype HLA-ABC 72, Biotest AG, 63303 Dreiech, Germany). If the data were inconclusive, DNA analyses were performed (Dynal Allset+ SSP, Dynal Biotech, Oslo).

If twins had the same HLA phenotype, the twins were presumed to be HLA identical, sharing 2 haplotypes. Twins with no identical HLA alleles were presumed to share 0 haplotypes. If twins were sharing one HLA allele of the A, $\mathrm{B}$ and $\mathrm{Cw}$ loci they were assumed to share 1 haplotype.

\section{Statistical Analysis}

The number of twins in the different haplotype sharing groups were tested to ascertain if the distribution HLA

Address for correspondence: Ingrid Louise Titlestad, Department of Clinical Immunology, Odense University Hospital, Denmark. Email: it@dadlnet.dk 
Table 1

Danish Twin Register

\begin{tabular}{lcccc}
\hline $\begin{array}{l}\text { No. of HLA } \\
\text { haplotypes shared }\end{array}$ & 2 & 1 & 0 & $p$ \\
\hline $\begin{array}{l}\text { No. of DZ } \\
\text { twin pairs (114) }\end{array}$ & 29 & 52 & 33 & 0.5605 \\
$\begin{array}{l}\text { Expected } \\
\text { No. of DZ female }\end{array}$ & 28.5 & 57 & 28.5 & \\
twin pairs (60) & 14 & 27 & 19 & 0.4884 \\
$\begin{array}{l}\text { Expected } \\
\text { No. of DZ male }\end{array}$ & 15 & 30 & 15 & \\
twin pairs (54) & 15 & 25 & 14 & \\
Expected & 13.5 & 27 & 13.5 & 0.8465 \\
\hline
\end{tabular}

haplotype sharing differed from the expected Mendelian $1: 2: 1$. The subjects were stratified by sex and compared using Chi-square test.

\section{Results}

The total of $114 \mathrm{DZ}$ twins were HLA-A, - B, (-Cw) typed and analyzed for shared alleles (see Table 1). Twenty-nine (expected 28.5) twin pairs had two HLA-haplotypes in common, 52 (expected 57) had one HLA-haplotype in common and 33 (expected 28.5) had zero HLA-haplotypes in common, and no significant difference from the Mendelian distribution was found. The same results were found when stratified for sex.

\section{$\overline{\text { Discussion }}$}

The $114 \mathrm{DZ}$ twins HLA-typed were enrolled without selection from a population based register. In this study design we have not been able to HLA type parents to test for identity-by-descent and thus had a risk of overestimating twins sharing haplotypes. With a large number of alleles and, assuming Hardy-Weinberg Equilibrium and random mating, the probability of identity-by-descent being significantly different from identity-by-state is low. With the null hypothesis being that DZ twins are more similar than sibs in general, this would not, however, alter the conclusions in any way.

Staub Nielsen et al. (1990) included 97 DZ twin pairs and found DZ twins of the same sex to have zero haplotypes in common less frequently than expected. DZ twins of different sex showed opposite characteristic, but only the distribution of 38 same sex twin pairs combined showed a significant departure from the Mendelian distribution.

Jawaheer et al. (1996) investigated a panel of $93 \mathrm{DZ}$ twin pairs discordant for rheumatoid arthritis. Their data showed a statistical difference in the distribution when compared to $128 \mathrm{sib}$ pairs. When stratifying for sex, only the female/female twin pairs (43) showed a significant
Table 2

Number of HLA Haplotypes Shared (From Winata et al. (1981)

\begin{tabular}{lcccc}
\hline $\begin{array}{l}\text { No. of HLA } \\
\text { haplotypes shared }\end{array}$ & 2 & 1 & 0 & $p$ \\
\hline $\begin{array}{l}\text { No. of DZ } \\
\text { twin pairs (71) }\end{array}$ & 22 & 39 & 10 & 0.0932 \\
$\begin{array}{l}\text { Expected } \\
\begin{array}{l}\text { No. of DZ female } \\
\text { twin pairs (42) }\end{array}\end{array}$ & 17.75 & 35.5 & 17.75 & \\
$\begin{array}{l}\text { Expected } \\
\begin{array}{l}\text { No. of DZ male } \\
\text { twin pairs (29) }\end{array}\end{array}$ & 10.5 & 19 & 9 & 0.4558 \\
Expected & 7.25 & 14.5 & 10.5 & \\
\hline
\end{tabular}

increase $(p<0.025)$ in haplotype sharing compared to the expected, and DZ twins of opposite sex had the distribution 1:2:1 of haplotype sharing. The data are at risk of being biased due to prior selection. Rheumatoid arthritis is known to be associated with certain HLA alleles.

Winata et al. (1981) investigated 71 pairs of same-sex DZ twins initially comparing within-pair differences for plasma total-, free- and esterified cholesterol, and triglyceride with the number of HLA haplotypes the twins had in common. In these data sets male/male twins showed statistical increased sharing of both HLA haplotypes compared to the expected (see table 2).

In conclusion, earlier papers on DZ HLA-haplotyping have enrolled a limited number of twin pairs, and the data could have been biased due to prior selection of the twins. The latter papers do also show diametrically opposing data, and the findings are probably accidental. Our data including 114 same sex DZ twins show that DZ twins are not more similar than sibs in general from different pregnancies.

\section{References}

Jawaheer, D., MacGregor, A. J., Gregersen, P. K., Silman, A. J., \& Ollier, W. E. (1996). Unexpected HLA haplotype sharing in dizygotic twin pairs discordant for rheumatoid arthritis. Journal of Medical Genetics, 33(12), 1015-1018.

Kyvik, K. O., Green, A., \& Beck-Nielsen, H. (1995). The new Danish Twin Register: Establiment and analysis of twinning rates. International Journal of Epidemiology, 23(3), 589-596.

Staub Nielsen, L., Eiberg, H., Fenger, K., \& Mohr, J. (1990). An MHC (HLA-A, -B, C2, BF, HLA-DR, GLO1) haplotype study of 497 Danish normal families with 190 children including 97 twin pairs. Tissue Antigens, 36(4), 141-148.

Winata, S. S., Beigel, A. A., Kang, K. W., Harmath, F., \& Christian, J. C. (1981). A search for stratification-free association between plasma lipids and HLA using dizygotic twins. Acta Geneticae Medicae et Gemellologiae (Roma), 30(3), 213-216. 